\title{
Dentigerous Cyst in Association with Impacted Mesiodens in Non Syndromic Patient
}

\author{
Syed Mustansir Ul Hassnain ${ }^{1}$,Vidya Devichandavarkar ${ }^{2}$, Deepak Bhargava ${ }^{3}$, Mithilesh Mishra ${ }^{4}$, \\ Ritika Sharma $^{5}$, Neha Singh ${ }^{6}$
}

\begin{abstract}
The literal meaning of dentigerous is 'tooth bearing'. Dentigerous cysts are the second most common odontogenic cysts after radicular cysts, most commonly seen in association with the mandibular third molar, followed in frequency by maxillary canine, maxillary third molar, and rarely in relation to maxillary central incisor. Dentigerous cysts around supernumerary teeth, however, account for 5\% of all dentigerous cysts, with most developing around a mesiodens in the anterior maxilla. This article reports a rare and unusual case of a dentigerous cyst of the anterior maxilla involving a multiple inverted mesiodens crossing the mid line. The complications associated with inverted mesiodens include eruption disturbance of adjacent teeth, displacement \& rotation of the central incisors, diastema, root resorption, abnormal occlusion, cyst formation or nasal eruption of the mesiodens.
\end{abstract}

Keywords: dentigerous cyst, mesiodens, supernumerary teeth

\section{Introduction}

The term "dentigerous cyst" was coined by Paget in $1853^{1}$. Dentigerous cysts are one of the most prevalent types of odontogenic cysts, mostly associated with an embedded or un-erupted tooth.A dentigerous cyst is one which encloses the crown of an un-erupted tooth by expansion of its follicle and is attached to the neck.They are usually associated with an impacted tooth and develop after the complete formation of the crown. They most commonly involve the mandibular third molars or the maxillary canine, followed by the mandibular premolars. The involvement of incisors and supernumerary teeth are $\operatorname{rare}^{2}$.'Mesiodens' are the most common supernumerary teeth situated between the maxillary central incisors.Mesiodens is supernumerary tooth of anterior maxilla. It can be classified as eumorphic and dysmorphic. The eumorphic subclass resembles normal tooth in size and shape whereas dysmorphic have different shapes and sizes. These can be further classified as conical, tuberculate, supplemental and odontomas. Most of mesiodens are conical in shape with short complete root. These can be normally aligned or inverted or placed horizontally. Tuberculate mesiodens are barrel shaped with several cusp or tubercles and have incomplete or abnormal root and these rarely erupt in the oral cavity ${ }^{3}$. A radiograph of the dentigerous cyst shows a well-defined uni-locular radiolucency, often with a sclerotic border, surrounding the crown of an unerupted tooth.

\section{Case Report}

A 36 year old male patient visited the outpatient department complaining of pain and swelling in the upper anterior maxillary region since 6 months. Initially the swelling was small with occasional dull pain. The swelling and pain had gradually increased leading to discomfort. His systemic history and family history were not significant (Figure 1).

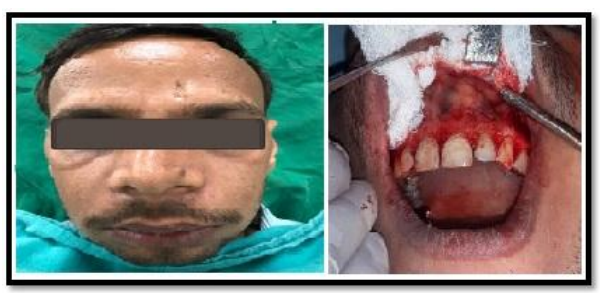

Figure 1

On routine hematological examination the random blood sugar, bleeding time and clotting time values were normal. There was no history of allergic symptoms. The patient had taken an unknown medication for the pain and swelling, but he had no relief.

Extra oral examination showed asymmetry of the face due to the swelling on the left side. The swelling was hard, diffuse, ovoid shape and tender, approximately measuring around $3 \mathrm{~cm} \times 2 \mathrm{~cm}$ extending from midline and left lateral wall of the nose, mildly obliterating the nasolabial fold and philtrum of the lip (Figure 1).

Intra-oral examination revealed a well-defined hard, tender and ovoid swelling involving labial aspect of right and left central incisors with labial cortical plate's expansion (Figure $1)$.

Orthopantomogram showed a well-defined and uni-locular radiolucent lesion approximately measuring $3 \times 3.5 \mathrm{~cm}$, attached to the crown of the unerupted inverted mesiodens in the right alveolar process of the anterior maxilla. The radiograph of the lesion revealed two impacted supernumerary teeth one with maxillary right central incisor and the other was in association with the roots of left maxillary central and lateral incisors and was associated with a well-defined radiolucent area in the anterior region of the maxilla crossing the midline (figure2). Non contrast computed tomography in axial and coronal planes showed fluid filled uni-locular lesion along with crown of mesiodens in the maxillary alveolar process (Figure 3).

FNAC of the lesion was done revealing a straw colored glittering cystic fluid and on cytopathological examination nonspecific inflammatory cells were seen (Figure 4). 


\section{International Journal of Science and Research (IJSR) \\ ISSN (Online): 2319-7064}

Index Copernicus Value (2015): 78.96 | Impact Factor (2015): 6.391

$H \&$ E stained section shows cystic lumen lined by thin epithelium resembling reduced enamel epitheliumand fibrous wall. The epithelium showed 2-3 layer thickness of cuboidal cells. The fibrous wall showed collagen bundles with interspersed fibroblasts. Overall clinicopathological features were suggestive of dentigerous cyst (Figure 5).

Under local anesthesia buccal crevicular incision was given from right to left maxillary canine region. Full buccal mucoperiosteal flap was reflected, lesion was exposed and mesiodens and whole of the cystic lining was removed with surgical curette (Figure 1).

\section{Discussion}

Dentigerous cyst is one that encloses the crown of an unerupted tooth by expansion of its follicle, and is attached to its neck ${ }^{4}$.This is the most common type of developmental odontogenic cyst, making up about $20 \%$ of all epithelium lined cysts of the jaws. The pathogenesis of this cyst is uncertain, but apparently it develops by accumulation of fluid between the reduced enamel epithelium and the tooth crown $^{5}$. Attachment of the cyst wall to the neck of the associated tooth is an essential feature, and microscopically, the cyst lining it has been suggested that the dentigerous cysts may be of extra follicular or intra follicular origin and that of intra follicular origin may develop by accumulation of fluid either between the reduced enamel epithelium and the enamel, or within the enamel organ itself ${ }^{4}$.

Dentigerous cyst is always associated initially with the crown of an impacted, embedded or unerupted tooth. A dentigerous cyst may also be found enclosing a complex compound odontoma or involving a supernumerary tooth. The most common sites of this cyst are the mandibular and maxillary third molar and maxillary cuspid areas since these are the most commonly impacted teeth ${ }^{6}$.

Dentigerous cysts are usually asymptomatic but may grow to a large size and may produce an intra-oral swelling and occasionally an extra-oral swelling, or may displace the tooth with which they are associated, or rarely cause resorption of adjacent tooth roots ${ }^{7}$. They usually present in the second or third decades of life and are rarely seen during childhood $^{8}$.

In our case, the dentigerous cyst was due to a supernumerary tooth/mesiodens. Stafne was the first to describe dentigerous cysts associated with supernumerary teeth, registering an incidence of $5.5 \%$ in 200 dentigerous cysts ${ }^{9}$. The etiology of supernumerary teeth is unknown. Mesiodens may be single or multiple, erupted or impacted, and are rarely seen associated with a dentigerous cyst. The direction of the crown of the mesiodens may be normal, inverted, or horizontal. Mesiodens are known to have a cone-shaped crown and a short root as seen in our patient. Radiological examinations are indicated for the diagnosis of mesiodens. Their location, number, direction of the crown, influence on the adjacent teeth, resorption of the adjacent roots, and formation of a dentigerous cyst should be carefully evaluated. Most mesiodens are located palatal to the permanent incisors. Only a few lie in the dental arch or labial to the permanent incisors. Resorption of the adjacent roots by mesiodens or its cyst is a rare complication ${ }^{10}$. In our patient, resorption of the roots was not observed.Jiang et al. literature review spanning from 1988 to 2011 discloses the publication of 16 cases of dentigerous cysts associated to supernumerary teeth in the premaxilla ${ }^{11}$.The mentioned predominance of follicular cysts associated to supernumerary teeth varies between 1 and 9.9\%. Likewise, a retrospective study conducted by Zhang et al. shows that only $11(1 \%)$ out of 2082 dentigerous cysts were related to an impacted supernumerary tooth, however, the connection with third molars (98\%), was the most common situation. On the other hand, Hurlen and Humerfelt found a correlation of $7 \%$ and Asaumi et al. stated that dentigerous cysts associated to supernumerary teeth account for $11 \%$ of the cases. A study by Lin et al. showed that the connection between dentigerous cysts and supernumerary teeth was of $13 \%{ }^{12}$

Radiographically, the dentigerous cyst typically appears as a well-circumscribed, uni-locular, usually symmetric radiolucency around the crown of an impacted tooth. An important diagnostic point is that this cyst attaches at the cementoenamel junction. The internal aspect of the cyst is completely radiolucent except for the crown of the involved tooth. One of the most difficult conditions to distinguish in the differential diagnosis is hyperplastic follicle. Other conditions that must be excluded in the diagnosis are odontogenic keratocyst, ameloblastic fibroma, and cystic ameloblastoma. Water's view, panoramic, and skull radiography are simple and inexpensive preliminary diagnostic methods.

Histological examination usually shows a thin fibrous cyst wall which, being derived from dental follicle, consists of young fibroblasts widely separated by stroma and ground substance rich in acid mucopolysaccharide. The epithelial lining, which is in fact reduced enamel epithelium, consists of 2-4 cell layers of flat or cuboidal cells. Characteristically, the epithelial lining is not keratinized. Discontinuities in the epithelial lining may be seen in the presence of an intense inflammatory infiltrate in the adjacent capsule, or, as suggested by Toller (1966), through partial adherence to enamel. Sometimes, the superficial layer of the epithelial lining is low columnar and retains the morphology of the ameloblast layer which, of course, it originally was.Localized proliferation of the epithelial lining may occur in response to inflammation. Occasional bud-like thickenings of the epithelium may be seen in the absence of inflammation and sometimes there may be budding of the basal cells into the fibrous capsule.Hyaline bodies (Rushton, 1955) are sometimes seen ${ }^{1}$.

The treatment modalities range from enucleation to marsupialization.Complete enucleation of cyst along with extraction of the tooth forms mainstay of treatment for dentigerous cyst associated with supernumerary tooth.Pathological fractures, ameloblastoma, squamous cell carcinoma, mucoepidermoid carcinoma are frequent other complications associated with dentigerous cyst ${ }^{13}$.

\section{Volume 6 Issue 7, July 2017 www.ijsr.net}




\section{International Journal of Science and Research (IJSR) \\ ISSN (Online): 2319-7064}

Index Copernicus Value (2015): 78.96 | Impact Factor (2015): 6.391

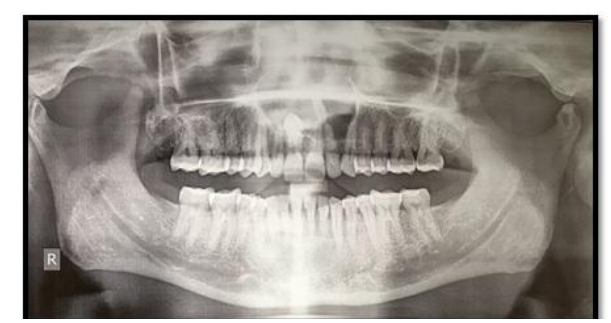

Figure 2

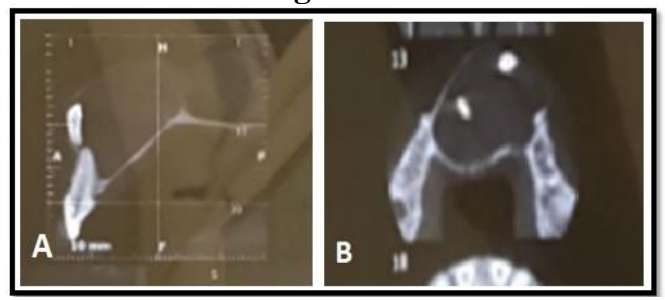

Figure 3

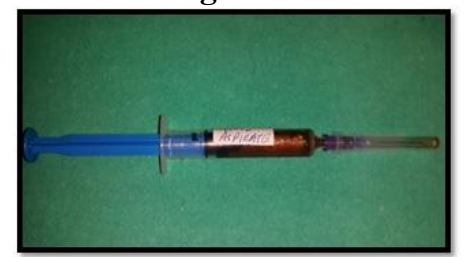

Figure 4

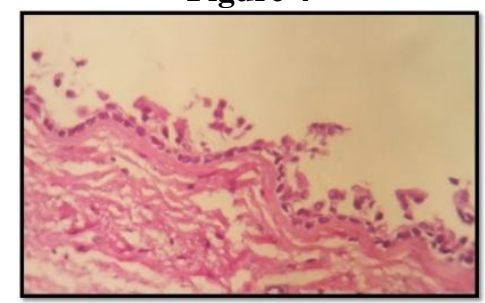

Figure 5

\section{References}

[1] Deepak JHH, Sana RM, Vinothini G, Thanigainathan R, Kandasamy M. Maxillary Dentigerous Cyst with Impacted Mesiodens - A Case Report. J Adv Med Dent Scie Res 2015; 3(3):119-22.

[2] More CB, Patel HJ. Dentigerous Cyst Associated With Mesiodens-A Rare Case Report. International Journal of Dental Clinics. 2011 Mar 31; 3(1).

[3] Saddiwal R, Hebbale M, Sane V. Dentigerous cyst associated with an impacted inverted supernumerary tooth in the maxillary anterior region-a case report.RSBO. 2015 Apr-Jun; 12(2):220-4.

[4] Shear M, Speight P. Cysts of the oral and maxillofacial regions. John Wiley \& Sons; 2008 Apr 15.

[5] Neville BW, Damm DD, Chi AC, Allen CM. Oral and Maxillofacial Pathology. Elsevier Health Sciences; 2015.

[6] Rajendran R. Shafer's textbook of Oral Pathology. Elsevier India; 2009.

[7] Scully C, Flint S, Porter SR, Moos K, Bagan J. Oral and Maxillofacial Diseases. CRC Press; 2010 Jun 15.

[8] González Navarro B, Jané Salas E, TeixidorOlmo I, Font i Muñoz A, Juarez Escalona I, LópezLópez J. Maxillary dentigerous cyst and supernumerary tooth. Is it a frequent association? Oral Health and Dental Management, 2014;13:127-31

[9] Jiang Q, Xu GZ, Yang C, Yu CQ, He DM, Zhang ZY. Dentigerous cysts associated with impacted supernumerary teeth in the anterior maxilla. Experimental and therapeutic medicine. 2011 Sep1; 2(5):805-9.

[10] Baranwal HC, Verma PK, Dwivedi CD, Srivastava R. Dentigerous cyst associated with an impacted maxillary mesiodens. European Journal of General Dentistry. 2012; 1(1):50.

[11] Gulses A, Karacayli U, Koymen R. Dentigerous cyst associated with inverted and fused supernumerary teeth in a child: a case report. Oral Health Dent Manag 2009;8:38-41

[12] Jiang Q, Xu GZ, Yang C, Yu CQ, He DM, Zhang ZY. Dentigerous cysts associated with impacted supernumerary teeth in the anterior maxilla. Experimental and therapeutic medicine. 2011 Sep1; 2(5):805-9.

[13] Narsapur SA, Choudhari S. Dentigerous cyst with atypical mesiodens: an unusual clinical case report RSBO (Online). 2015 Jun; 12(2):220-4. 